\title{
Improving the accuracy of detecting the edges of texture objects in remote sensing images
}

\author{
E V Medvedeva ${ }^{1}$, A I Evdokimova ${ }^{1}$ \\ ${ }^{1}$ Vyatka State University, Moskovskaya str., 36, Kirov, Russia, 610000 \\ e-mail: emedv@mail.ru, alenaevdokimova0@gmail.com
}

\begin{abstract}
The authors offer a method for detecting the edges of texture objects in remote sensing images. This method is based on the evaluation of textural and brightness attributes. It is proposed to use transition probabilities for three-dimensional Markov chains with two states as texture features, averaged within a sliding window. It makes possible to improve the detection accuracy of texture objects on multichannel or multi-time snapshots. To reduce the computational resources, it is proposed to determine the signs by the bit planes of the senior, most informative digits of the digital image. The simulation results confirm the effectiveness of the proposed method.
\end{abstract}

\section{Introduction}

Multispectral remote sensing images are of high spatial resolution and have rich information about texture. Information about the texture allows to determine the types of the earth's surface (rocks, soil, vegetation, water, etc.), to estimate the area of flooding, land use, forestry, wildfires, oil pollution, ice, etc.

The ability to detect, identify, and classify texture objects is based on the fact that objects of different types reflect and absorb electromagnetic radiation in different wavelengths in different ways. When combining different channels, one can find out information about rocks, vegetation cover, state of reservoirs, types of agricultural crops, etc. For example, in the visible red spectral channel (wavelength $0.63-0.69 \mu \mathrm{m}$ ), it is possible to distinguish many plant varieties, boundaries of the soil and geological delineation (deposits of the ore body, oil fields) [1,2]. One can track the dynamics for several pictures of the same area, taken at different times, for example changing the size of the area of the segments corresponding to oil pollution, or changing the forest area.

The basic operation for detecting the edges of texture objects is segmentation, the quality of which is determined by the effectiveness of the selection of a textural feature. There are various approaches to the selection of textures. They are [3-9]:

- based on the calculation of the statistical characteristics of the gradient, density of differences, adjacency matrices of brightness levels, etc.,

- based on the description of textures as primitives with some properties (average intensity of elements, perimeter, area, orientation, etc.) and comparing them with a template for each texture,

- based on signal processing algorithms for extracting texture features (discrete cosine and wavelet transforms),

- based on models, including the autoregression model, Markov random fields, fractal methods, etc. 
One of the new approaches in the selection of textures is the joint use of spectral and textural features $[10,11]$.

Many of these methods require large computational resources and do not accurately separate the attribute space. The quality of the selection of texture objects depends on the choice of the most appropriate characteristics of the task and approaches to the development of algorithms. In addition, as multispectral images have a high spatial resolution, an important factor in the detection of textures is to reduce the computational complexity of algorithms. In this regard, the use of known methods is not always advisable.

As the texture regions on images occupy extended space with homogeneous statistical characteristics and different for different regions, the method based on the mathematical apparatus of Markov chains [12] is very effective in this case. Its complexity depends on the dimension of the transition probability matrices.

Works [13,14] propose to solve the computational complexity reduction problem using representation of $\mathrm{g}$ - bit digital images (DI) as a set of $\mathrm{g}$ - bit binary images (BBI) and approximate them with Markov chains with two states and $2 \times 2$ transition probability matrices. Assuming that the most informative are the binary images belonging to the senior bits of DI, it is proposed to use one of the BBIs of the senior bits of DI to select textures. This solution reduces computational resources in the allocation of textures.

When using the mathematical apparatus of Markov chains, textural features are probabilistic characteristics between elements of images. Works [14-16] present the method of texture segmentation of DI based on a two-dimensional Markov chain with two states. With probabilities of transitions between elements different by 0.15 for different texture areas, the segmentation error did not exceed $6 \%$.

Considering that there is a large statistical dependence between separate areas of images taken in different spectral ranges (channels), it is proposed to use the nature of the statistical connection not only between the elements inside the DI, but also the inter-channel DI. The use of the transition probability for three-dimensional Markov chains as a textural attribute improves the accuracy of prediction of image elements and, accordingly, the quality of the selection of textures. Also, an approach based on three-dimensional Markov chains can be applied to detect changed texture regions in time-varying DIs.

Real remote sensing DIs contain objects in which the pixel brightness changes slightly (water bodies, fields of different crops, etc.). Therefore, to improve the quality of detection of texture objects for which the probabilistic characteristics between the elements are close to unity, it is advisable to take into account the brightness characteristics determined by BBI of the senior bits DI.

The aim of the article is to develop a method of segmentation of multi-channel digital images with high spatial resolution based on the mathematical apparatus of three-dimensional Markov chains, which allows to increase the accuracy of detecting the edges of texture objects while reducing computational resources.

\section{Mathematical model of the texture image}

A special case of multichannel images can be considered color RGB images. Therefore, the proposed method will be considered for the case of three-component DI with R, G, and B color channels. Each RGB color component of an image is a g-digit digital halftone image. There is a significant statistical relationship between the elements of individual DI areas belonging to different color channels. For example, objects of yellow color are well expressed on green and red components, and objects of white color are seen on all three components. Therefore, taking into account the statistical connection between the elements inside DI and between individual color channels, we can assume that RGB images allow them to be approximated by a three-dimensional Markov chain with several states, and BBI with a three-dimensional Markov chain with two states and horizontal transition matrixes ${ }^{1} \boldsymbol{\Pi}=\left\|{ }^{1} \pi_{i j}^{(l)}\right\|_{2 \times 2}$, vertical ${ }^{2} \boldsymbol{\Pi}=\left\|{ }^{2} \pi_{i j}^{(l)}\right\|_{2 \times 2}$ and ${ }^{4} \boldsymbol{\Pi}=\left\|{ }^{4} \pi_{i j}^{(l)}\right\|_{2 \times 2}$ 
between channels. The introduction of the inter-channel matrix ${ }^{4} \boldsymbol{\Pi}=\left\|{ }^{4} \pi_{i j}^{(l)}\right\|_{2 \times 2}$ will make it possible to get rid of the segmentation of the separate components and identify the changed areas in the images.

Figure 1 shows a fragment of a three-dimensional binary Markov field corresponding to a fragment of an BBI of a two-channel DI.

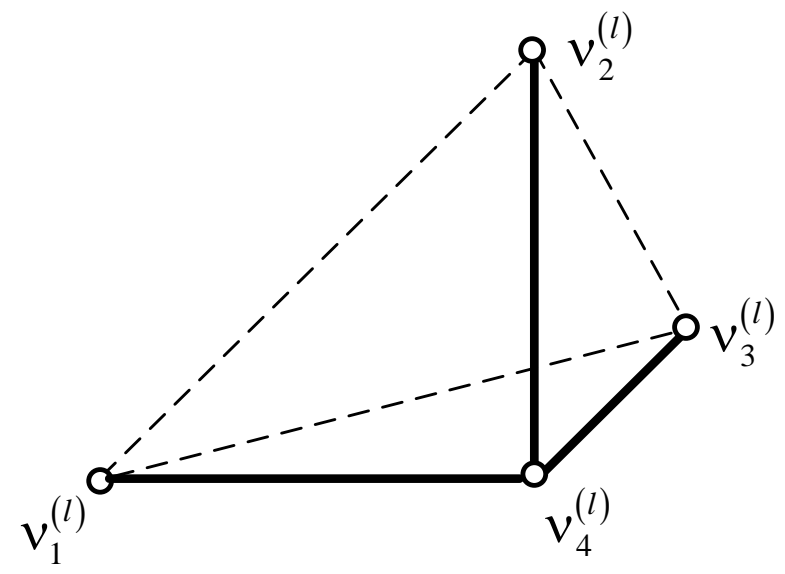

Figure 1. Fragment of a three-dimensional binary Markov field.

The amount of information in the element $v_{4}^{(l)}$ relative to the elements of the nearest neighborhood $\Lambda_{i, j, k}=\left\{v_{1}^{(l)}, v_{2}^{(l)}, v_{3}^{(l)}\right\}$, in accordance with the mathematical model of the three-dimensional random Markov process presented in [13], is determined by the formula:

$$
I\left(v_{4}^{(l)} \mid v_{1}^{(l)}, v_{2}^{(l)}, v_{3}^{(l)}\right)=-\log \frac{\prod_{i=1}^{3} w\left(v_{4}^{(l)} \mid v_{i}^{(l)}\right) w\left(v_{4}^{(l)} \mid v_{1}^{(l)}, v_{2}^{(l)}, v_{3}^{(l)}\right)}{\prod w\left(v_{4}^{(l)} \mid v_{i}^{(l)}, v_{j}^{(l)}\right)},
$$

where $w\left(v_{4}^{(l)} \mid \square\right)$ is the probability density of transitions in Markov chains of various dimensions; the products in (1) are calculated for all possible non-coinciding combinations of various subscript indices.

The probability density of transitions in a three-dimensional binary Markov chain $w\left(v_{4}^{(l)} \mid \Lambda_{i, j . k}^{(l)}\right)$ can have the form:

$$
\begin{aligned}
& w\left(v_{4}^{(l)} \mid \Lambda_{i, j, k}^{(l)}\right)=\sum_{i, \ldots, r=1}^{2} \pi\left(v_{4}^{(l)}=M_{i}^{(l)} \mid v_{1}^{(l)}=M_{j}^{(l)} ; v_{2}^{(l)}=M_{k}^{(l)} ;\right. \\
& \left.v_{3}^{(l)}=M_{r}^{(l)}\right) \times \delta\left(v_{1}^{(l)}-M_{j}^{(l)}\right) \delta\left(v_{2}^{(l)}-M_{k}^{(l)}\right) \delta\left(v_{3}^{(l)}-M_{r}^{(l)}\right) .
\end{aligned}
$$

where $\delta(\cdot)$ is the delta function.

The probabilities of the states $\pi_{\text {iiii }}^{(l)}$ of an element $v_{4}^{(l)}$ are determined by the argument of expression (1) and for various combinations of neighboring elements $\Lambda_{i, j, k}^{(l)}$ can be calculated using the formulae presented in Table 1 , where ${ }^{r} \pi_{i i}^{(l)}(r=\overline{1,7})$ are elements of transition probability matrices in onedimensional Markov chains with two states of three main - ${ }^{1} \Pi^{(l)},{ }^{2} \Pi^{(l)},{ }^{4} \Pi^{(l)}$ and four related matrices ${ }^{3} \boldsymbol{\Pi}^{(l)}={ }^{1} \boldsymbol{\Pi}^{(l)} \times{ }^{2} \boldsymbol{\Pi}^{(l)} ;{ }^{5} \boldsymbol{\Pi}^{(l)}={ }^{1} \boldsymbol{\Pi}^{(l)} \cdot{ }^{4} \boldsymbol{\Pi}^{(l)} ;{ }^{6} \boldsymbol{\Pi}^{(l)}={ }^{2} \boldsymbol{\Pi}^{(l)} \cdot{ }^{4} \boldsymbol{\Pi}^{(l)} ;{ }^{7} \boldsymbol{\Pi}^{(l)}={ }^{3} \boldsymbol{\Pi}^{(l)} \cdot{ }^{4} \boldsymbol{\Pi}^{(l)}$. 
Table 1. Expressions for calculating conditional probabilities.

\begin{tabular}{|c|c|c|}
\hline$v_{1} v_{2} v_{3} \rightarrow v_{4}$ & Expressions & \\
\hline $000 \rightarrow 0$ & $\pi_{i i i i}^{(l)}=\frac{{ }^{1} \pi_{i i}^{(l)} \cdot{ }^{2} \pi_{i i}^{(l)} \cdot{ }^{4} \pi_{i i}^{(l)} \cdot{ }^{7} \pi_{i i}^{(l)}}{{ }^{3} \pi_{i i}^{(l)} \cdot{ }^{5} \pi_{i i}^{(l)} \cdot{ }^{6} \pi_{i i}^{(l)}}$ & \\
\hline $001 \rightarrow 0$ & $\pi_{i i j i}^{(l)}=\frac{{ }^{1} \pi_{i i}^{(l)} \cdot{ }^{2} \pi_{i i}^{(l)} \cdot{ }^{4} \pi_{i j}^{(l)} \cdot{ }^{7} \pi_{i j}^{(l)}}{{ }^{3} \pi_{i i}^{(l)} \cdot{ }^{5} \pi_{i j}^{(l)} \cdot{ }^{6} \pi_{i j}^{(l)}}$ & \\
\hline $111 \rightarrow 0$ & $\pi_{j j j i}^{(l)}=\frac{{ }^{1} \pi_{i j}^{(l)} \cdot{ }^{2} \pi_{i j}^{(l)} \cdot{ }^{4} \pi_{i j}^{(l)} \cdot{ }^{7} \pi_{i j}^{(l)}}{{ }^{3} \pi_{i i}^{(l)} \cdot{ }^{5} \pi_{i i}^{(l)} \cdot{ }^{6} \pi_{i i}^{(l)}}$ & (3) \\
\hline $000 \rightarrow 1$ & $\pi_{i i i j}^{(l)}=\frac{{ }^{1} \pi_{i j}^{(l)} \cdot{ }^{2} \pi_{i j}^{(l)} \cdot{ }^{4} \pi_{i j}^{(l)} \cdot{ }^{7} \pi_{i j}^{(l)}}{{ }^{3} \pi_{i i}^{(l)} \cdot{ }^{5} \pi_{i i}^{(l)} \cdot{ }^{6} \pi_{i i}^{(l)}}$ & \\
\hline $001 \rightarrow 1$ & $\pi_{i i j j}^{(l)}=\frac{{ }^{1} \pi_{i j}^{(l)} \cdot{ }^{2} \pi_{i j}^{(l)} \cdot{ }^{4} \pi_{i i}^{(l)} \cdot{ }^{7} \pi_{i i}^{(l)}}{{ }^{3} \pi_{i i}^{(l)} \cdot{ }^{5} \pi_{i j}^{(l)} \cdot{ }^{6} \pi_{i j}^{(l)}}$ & \\
\hline $111 \rightarrow 1$ & $\pi_{j j j j}^{(l)}=\frac{{ }^{1} \pi_{i i}^{(l)} \cdot{ }^{2} \pi_{i i}^{(l)} \cdot{ }^{4} \pi_{i i}^{(l)} \cdot{ }^{7} \pi_{i i}^{(l)}}{{ }^{3} \pi_{i i}^{(l)} \cdot{ }^{5} \pi_{i i}^{(l)} \cdot{ }^{6} \pi_{i i}^{(l)}}$ & \\
\hline
\end{tabular}

\section{Texture 3D Segmentation Algorithm}

Allocation of extended textures in multichannel (or multi-temporal) DI was reduced to calculating estimates of transition probabilities horizontally ${ }^{1} \hat{\pi}_{i i}^{(l)}$, vertically ${ }^{2} \hat{\pi}_{i i}^{(l)}$, between channels ${ }^{4} \hat{\pi}_{i i}^{(l)}$ and estimation $\hat{\pi}_{\text {iiii }}^{(l)}$ using formulas (3), as well as estimating brightness in BBI of the senior, most informative DI digits.

Taking into account the local changes in the probability and brightness characteristics on multichannel images, a three-dimensional sliding window was used for calculation.

The texture features were the average estimates corresponding to the central element of the window - $\tilde{\pi}_{i i i i}^{(l, r, k)}$ of probability of transitions in the three-dimensional Markov chain and the brightness $\tilde{L}^{(l, r, k)}$ of the BBI:

$$
\begin{aligned}
& \tilde{\pi}_{i i i i}^{(l, r, k)}=\frac{1}{m \times n} \sum_{r=1}^{m} \sum_{k=1}^{n} \hat{\pi}_{i i i i}^{(l, r, k)} . \\
& \tilde{L}^{(l, r, k)}=\frac{1}{m \times n} \sum_{r=1}^{m} \sum_{k=1}^{n} \hat{L}^{(l, r, k)} .
\end{aligned}
$$

The pixel belonging to one or another texture object in the image of the k- channel was carried out on the basis of the analysis of the texture feature histogram. The number of peaks in the histogram corresponded to the number of texture objects with different probabilistic characteristics. The threshold value was chosen as the minimum value between two adjacent peaks of the histogram. When 
evaluation $\tilde{\pi}_{i i i i}^{(l, r, k)}$ was close to 1 , the averaged brightness $\tilde{L}$ was additionally used to decide whether a pixel belongs to one or another object. Each texture object was assigned its own label.

The authors combined images of different channels in order to increase the information content of texture objects.

\section{Experimental results}

Artificial and real DI were used for experimental studies. Artificial binary images were generated by a given markup using a mathematical model based on a two-dimensional Markov chain and the algorithm given in [13]. The images were formed in such a way that they contained areas with coincident and different statistical characteristics for different channels.

To assess the accuracy of the selection of the edges of texture objects, the authors compared the segmented image with the ideal markup and then calculated the number of erroneously segmented elements:

$$
E S E=\frac{1}{h \cdot w} \sum_{i=1}^{h} \sum_{j=1}^{w} F(i, j)
$$

where $h, w$ are image height and width; $F$ is a value that is equal to zero when the image element is segmented correctly, and equal to 1 if otherwise.

Examples of the results of segmentation of artificial images are shown in Figure 2: (a) an image containing two areas with different statistical characteristics; (b) the result of a segmentation algorithm based on a three-dimensional Markov chain with a difference between the probabilities $\Delta \tilde{\pi}_{i i i i}$ of segments in 0.25; (в) an image with three objects; (d) the result of a segmentation algorithm based on an estimation of probability $\tilde{\pi}_{i i i i}^{(l, r, k)}$ and brightness $\tilde{L}$.

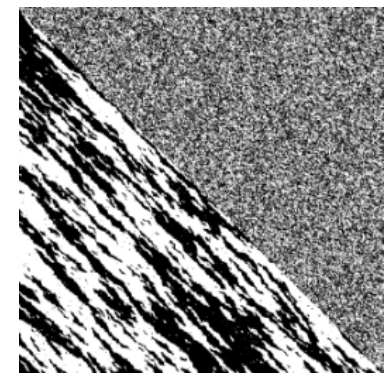

a)

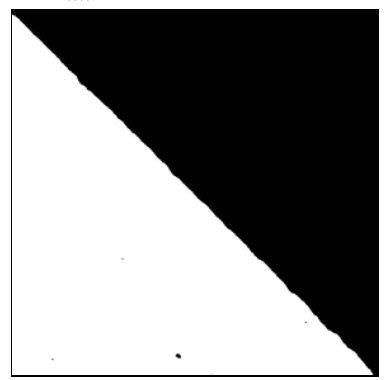

b)

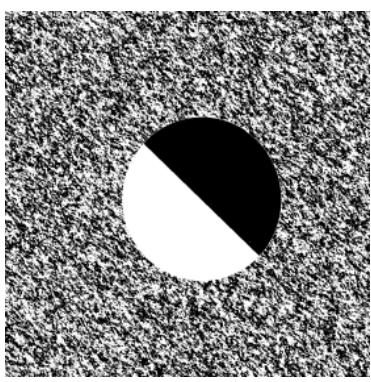

c)

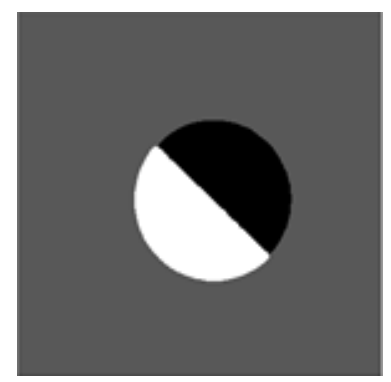

d)

Figure 2. Results of the segmentation of artificial images.

Table 2 presents the estimates of the ESE criterion for segmented artificial images containing two texture regions (similar to Figure 2a). A sliding window of $11 \mathrm{x} 11$ size was used to calculate the probability estimations $\tilde{\pi}_{i i i i}$.

Table 2. Estimation of segmentation results by ESE criterion.

\begin{tabular}{llll}
\hline \multirow{2}{*}{$\begin{array}{l}\text { Value of transition probabilities } \\
\pi_{i i}={ }^{2} \pi_{i i}\end{array}$} & Value ESE, \% & \\
\cline { 3 - 4 } $\begin{array}{l}\text { in the first } \\
\text { segment }\end{array}$ & $\begin{array}{l}\text { in the second } \\
\text { segment }\end{array}$ & $\begin{array}{l}\text { Algorithm based on a two- } \\
\text { dimensional Markov chain }\end{array}$ & $\begin{array}{l}\text { Algorithm based on a three- } \\
\text { dimensional Markov chain }\end{array}$ \\
\hline 0.5 & 0.95 & 0.32 & 0.21 \\
0.6 & 0.85 & 0.79 & 0.29 \\
0.7 & 0.95 & 0.54 & 0.28 \\
0.8 & 0.9 & 6.31 & 5.88 \\
\hline
\end{tabular}

Three-dimensional segmentation for all values of the transition probabilities gave a segmentation error less than segmentation based on two-dimensional Markov chains. The worst segmentation result 
was obtained when the difference between the probabilities of the segments was 0.1 . As this difference increases, the segmentation error is a fraction of a percent.

Table 3 presents the estimates of the ESE criterion for segmented binary images, similar to Fig. 2c. Three-dimensional segmentation was performed on the basis of estimates of probability and brightness characteristics within a sliding window of $11 \times 11$.

Table 3. Estimation of segmentation results by ESE criterion.

\begin{tabular}{lll}
\hline \multirow{2}{*}{$\begin{array}{l}\text { Probabilities between elements in the texture } \\
\text { area }{ }^{1} \pi_{i i}={ }^{2} \pi_{i i}\end{array}$} & Without brightness & WSE, $\%$ \\
\hline 0.6 & 8.3182 & 0.61 \\
0.7 & 8.285 & 0.5738 \\
0.8 & 8.1621 & 0.4429 \\
\hline
\end{tabular}

Accounting for the brightness of the image in a given example allowed to reduce the segmentation error by up to 18 times.

An example of real image remote sensing segmentation, containing four types of objects (urban buildings, forest and fields planted with different crops) is shown in Figure 3: (a) - RGB image; (b) and (c) - BBI of the 7th category of channels R and B, which were used to select the edges of texture objects; (d) and (d) are the results of two-dimensional segmentation of the image of the $\mathrm{R}$ and $\mathrm{B}$ channels, respectively; (e) - the result of three-dimensional segmentation of channel $\mathrm{R}$ relative to channel B.

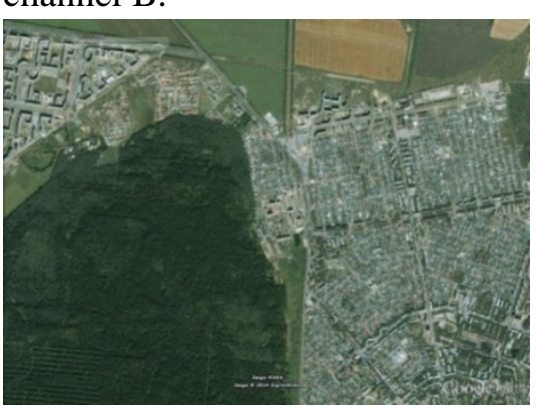

a)

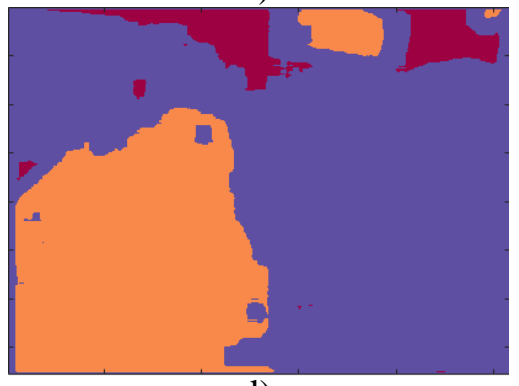

d)

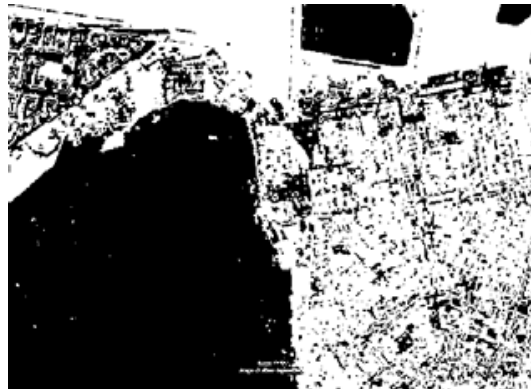

b)

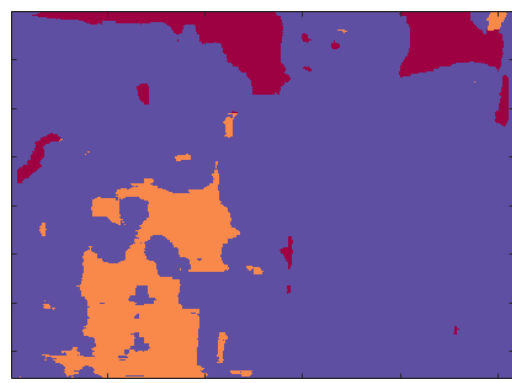

e)

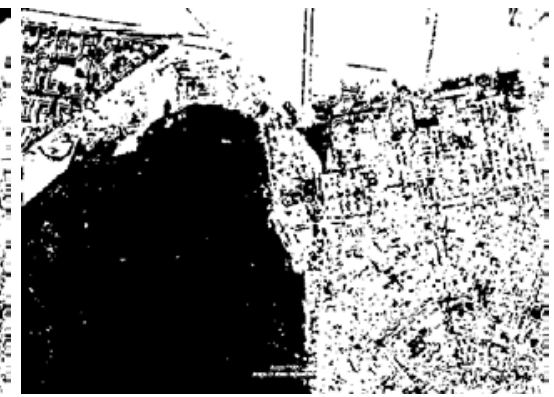

c)

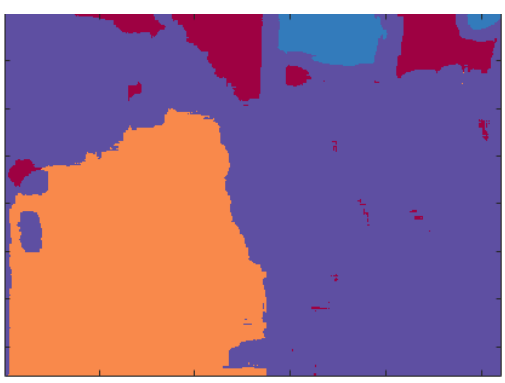

f)

Figure 3. The result of detecting the edges of texture objects in the remote sensing image.

Two-dimensional segmentation in the images of the $\mathrm{R}$ and $\mathrm{B}$ channels allowed to detect only 3 objects. The introduction of the matrix of probabilities of transitions between color components ${ }^{4} \boldsymbol{\Pi}=\left\|{ }^{4} \pi_{i j}^{(l)}\right\|_{2 \times 2}$ allowed to get rid of the segmentation of the separate components and to detect another object. The proportion of erroneously segmented elements is $7.8 \%$. 
Figure 4 shows an example of detecting texture objects on multi-temporal images: (a) and (b) multi-temporal images; (c) - the result of the detection of a modified object (felled forest) by a threedimensional segmentation algorithm; (d) - the combined image of the modified object with the original one (a).

a)

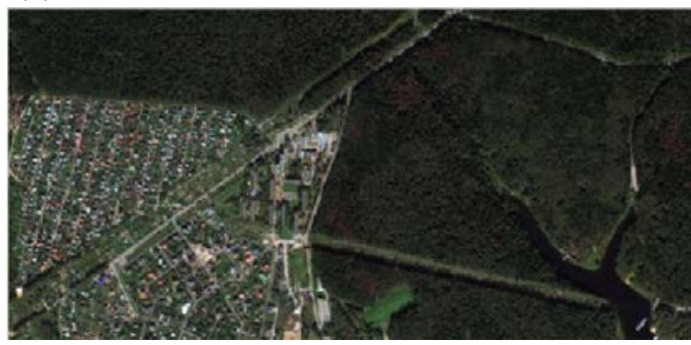

c)

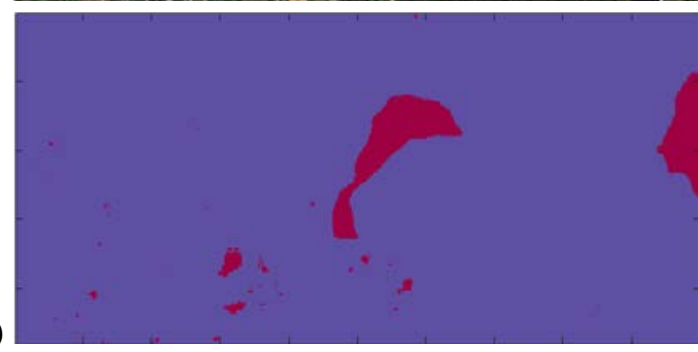

b)

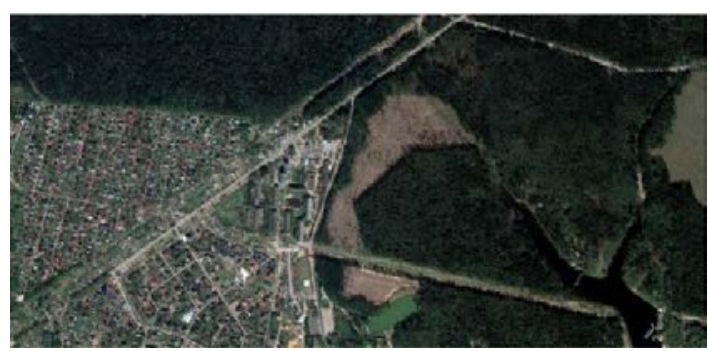

d)

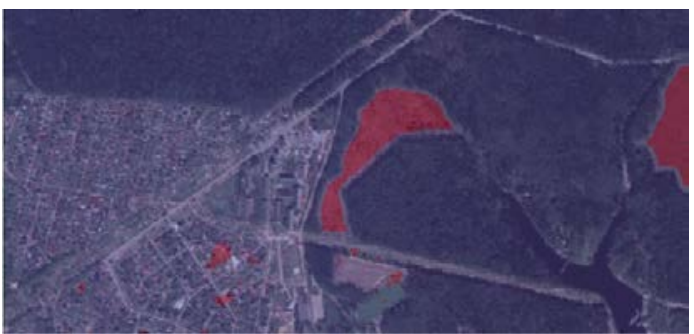

Figure 4. The result of detecting the edges of the modified texture objects in multi-time shots.

\section{Conclusion}

The developed segmentation method, based on an estimate of transition probabilities for threedimensional Markov chains and luminosity, improved the accuracy of detecting the edges of extended texture objects on multichannel and time-varying images compared to the two-dimensional segmentation algorithm proposed in [14]. In addition, the evaluation of texture features by bit binary images allows to reduce computational resources for the implementation of the segmentation algorithm.

\section{References}

[1] Schovengerdt R A 2010 Remote sensing. Models and methods of image processing (Moscow: Technosphere) p 594

[2] Ivanov E S 2016 Some applications of remote sensing image segmentation Modern problems of remote sensing of the Earth from space 13(1) 105-116

[3] Gonzalez R, Woods R 2012 Digital Image Processing (Moscow: Technosphere) p 1104

[4] Haralick R M 1979 Statistical and structural approaches to texture Proceedings of the IEEE 67(5) 786-804

[5] Li M, Zang S, Zhang B, Li S and Wu C A 2014 Review of Remote Sensing Image Classification Techniques: the Role of Spatio-contextual Information European Journal of Remote Sensing 47 389-411

[6] Borne F 2017 Texture-based classification for characterizing regions on remote sensing images Journal of Applied Remote Sensing 11(3) 16

[7] Su W, Zhang C, Yang J, Wu H, Deng L, Ou W, Yue A and Chen M 2012 Analysis of wavelet packet and statistical textures for object-oriented classification of forest-agriculture ecotones using SPOT 5 imagery International Journal of Remote Sensing 33(11) 3557-3579

[8] Plotnikov D E, Kolbudaev P A and Bartalev S A 2018 Identification of dynamically homogeneous areas with time series segmentation of remote sensing data Computer Optics 42(3) 447-456 DOI: 10.18287/2412-6179-2018-42-3-447-456

[9] Blokhinov Yu B, Gorbachev V A, Rakutin Yu O and Nikitin A D 2018 A real-time semantic segmentation algorithm for aerial imagery Computer Optics 42(1) 141-148 DOI: 10.18287/ 2412-6179-2018-42-1-141-148 
[10] Yuan J, Wang D L 2014 Remote sensing image segmentation by combining spectral and texture features IEEE Transactions on geoscience and remote sensing 52(1) 16-24

[11] Zhang J, Gao Y W, Feng S W, Chen Z H and Yuan Y B 2015 Image segmentation with texture clustering based JSEG Proc. International Conference on Machine Learning and Cybernetics (ICMLC) 2 599-603

[12] Stan Z L 2009 Markov Random Field Modeling in Image Analysis (Springer-Verlag London Limited) p 569

[13] Petrov E P, Trubin I S, Medvedeva E V and Smolskiy S M 2013 Mathematical Models of VideoSequences of Digital Half-Tone Images Integrated models for information communication systems and net-works: design and development 207-241

[14] Medvedeva E V, Kurbatova E E 2015 Image Segmentation Based on Two-Dimensional Markov Chains Computer Vision in Control Systems-2. Innovations in Practice (Springer International Publishing Switzerland) 277-295

[15] Medvedeva E V, Kurbatova E E 2017 A combined algorithm of isolation texture areas in noisy images 6th Mediterranean Conference on Embedded Computing 155-158

[16] Medvedeva E V, Kurbatov E E and Okulova A A 2017 Textural segmentation of noisy images of the Earth's surface Modern problems of remote sensing of the Earth from space 14(7) 20-28 